Am. J. Trop. Med. Hyg., 103(3), 2020, pp. 1204-1206

doi:10.4269/ajtmh.20-0551

Copyright $\odot 2020$ by The American Society of Tropical Medicine and Hygiene

\title{
Temporal Change of SARS-CoV-2 in Clinical Specimens of COVID-19 Pneumonia Patients
}

\author{
Viravarn Luvira, ${ }^{1}$ Akanitt Jittmittraphap, ${ }^{2}$ Sant Muangnoicharoen, ${ }^{1}$ Nantarat Chantawat, ${ }^{3}$ Weena Janwitthayanan, ${ }^{3}$ and \\ Pornsawan Leaungwutiwong ${ }^{2 *}$ \\ ${ }^{1}$ Department of Clinical Tropical Medicine, Faculty of Tropical Medicine, Mahidol University, Bangkok, Thailand; ${ }^{2}$ Department of Microbiology and \\ Immunology, Faculty of Tropical Medicine, Mahidol University, Bangkok, Thailand; ${ }^{3}$ Tropical Medicine Diagnostic Reference Laboratory, Faculty of \\ Tropical Medicine, Mahidol University, Bangkok, Thailand
}

\begin{abstract}
The quality and type of specimen collection affect the sensitivity of real-time reverse transcriptase-PCR (rRT-PCR) for the diagnosis of SARS-CoV-2. In this report, the course over time of rRT-PCR for SARS-CoV-2 in 26 clinical specimens collected from the upper (nasopharyngeal and throat swabs) and lower (sputum) respiratory tracts of COVID19 cases with pneumonia was investigated along with the clinical course. The preliminary results revealed that higher SARS-CoV-2 RNA concentration and longer time for detection make self-collected sputum a preferable specimen for the diagnosis and follow-up of COVID-19 pneumonia. Self-collection of sputum can minimize the risk of unnecessary exposure to healthcare workers, preserve the shortage of personal protective equipment, and limit viral transmission to the environment.
\end{abstract}

In December 2019, an outbreak of COVID-19, caused by SARS-CoV-2, began in China and had spread as a global pandemic. ${ }^{1-3}$ Real-time reverse transcriptase-PCR (rRT-PCR) has been the standard test for the diagnosis of SARS-CoV-2 in the early stages of the COVID-19 pandemic. However, the quality and the timing of specimen collection affect the sensitivity of the test. ${ }^{4}$ To date, no consensus practical guideline for specimen collection exists. Nasopharyngeal swabs (NPS) and throat swabs (TS) are widely used for the diagnosis of COVID-19. Detection of SARS-CoV-2 in different sample types and evaluation of the rRT-PCR results over time during the clinical course of the disease are limited so far. Herein, we describe temporal changes in rRT-PCR results for SARS-CoV-2 in respiratory-tract specimens, both NPS/TS and sputum, along with the clinical course of COVID-19 pneumonia.

We analyzed 26 clinical specimens from three cases of COVID-19 pneumonia hospitalized at the Hospital for Tropical Diseases in Bangkok during a COVID-19 outbreak in Thailand between March 2020 and May 2020. Pneumonia was diagnosed based on clinical symptoms and chest radiography. COVID-19 was confirmed by rRT-PCR for SARS-CoV-2 in different specimen types from the same day on clinical diagnosis and during the hospital course of pneumonia cases, including NPS/TS collected in a single aliquot of viral transport medium (VTM) and self-collected sputum in a container without VTM. The rRT-PCR for SARS-CoV-2 (detection kit for novel coronavirus 2019-nCoV RNA; DaAn Gene Co., Ltd., Guangdong, China) and respiratory pathogen panel for the detection and identification of 26 pathogens (Allplex ${ }^{\mathrm{TM}} \mathrm{Re}$ spiratory Panel Assays; Seegene Inc., Seoul, South Korea) were performed at the Tropical Medicine Diagnostic Reference Laboratory of the Faculty of Tropical Medicine, Mahidol University, Bangkok. A cycle threshold value (Ct value) less than or equal to 40 is defined as positive. The study was approved by the Ethics Committee of the Faculty of Tropical Medicine, Mahidol University, Thailand.

\footnotetext{
*Address correspondence to Pornsawan Leaungwutiwong, Department of Microbiology and Immunology, Faculty of Tropical Medicine, Mahidol University, 420/6 Ratchawithi Rd., Ratchathewi, Bangkok 10400, Thailand. E-mail: pornsawan.lea@mahidol.ac.th
}

All COVID-19 pneumonia cases were admitted in isolation and were treated with a combination of favipiravir, chloroquine, and darunavir/ritonavir, or lopinavir/ritonavir according to the National Treatment Guidelines for COVID-19 at that particular time for at least 10 days. ${ }^{5}$ Antimicrobial agents were prescribed according to the physician's decision. All cases were in isolation until the symptoms were clear and all clinical specimens revealed negative for SARS-CoV-2. The clinical and laboratory characteristics of the COVID-19 patients are summarized in Table 1.

Interestingly, two of three COVID-19 cases were coinfected with Haemophilus influenzae. Coinfection with respiratory pathogens including bacteria, viruses, and fungus has been reported with the rate varying among the study population and method..$^{6-8}$ However, the types of coinfected pathogens, the pathogen interaction, and disease severity among SARSCoV-2-positive cases are unclear so far. Clinical and experimental studies to fill these clinical gaps are warranted.

The dynamics of SARS-CoV-2 RNA concentration in different types of clinical specimen from COVID-19 pneumonia patients during the clinical course are shown in Figure 1. The rRT-PCR technique was performed to detect the ORF1b and $\mathrm{N}$-gene regions of SARS-CoV-2, both regions were well correlated in all specimens (Figure $1 \mathrm{~A}$ and $\mathrm{B}$ ). The revolution in clinical and $r R T-P C R$ results of each patient was illustrated in Figure 1C-E. The overall trend of higher concentration of virus (indicated by lower Ct value) in sputum than NPS/TS from the same day was observed. Viral RNA could be detected for longer time in sputum (during $>2-6$ weeks) than swab specimens in two of three cases, with an obviously longer time in one case (Figure 1E), implying that the correct types of specimens from the anatomical site of pathology-sputum in pneumonia cases-are preferable. ${ }^{4}$ Although some COVID19 patients reported dry cough, expectoration can be achieved in all pneumonia cases without the induction of sputum. The higher positive rates of SARS-CoV-2 in sputum than TS on diagnosis have been recently reported; however, the data of dynamic changes of virus detection in sputum during the course of illness are limited. ${ }^{9-11}$ Importantly, the detection period of 2-5 weeks after the onset of symptoms (Figure 1C-E) in swab specimens in our study was longer than those reported recently in hospitalized COVID-19 patients, in which SARS-CoV-2 were positive in swabs mainly in the first week, but in sputum until the second and third weeks of 
TABLE 1

Clinical and laboratory characteristics of COVID-19 patients

\begin{tabular}{|c|c|c|c|}
\hline & Case $1^{*}$ & Case 2 & Case 3 \\
\hline Age (years), gender & 30 , male & 47 , male & 68 , male \\
\hline Probable source of infection & $\begin{array}{l}\text { Local transmission, public } \\
\text { transport }\end{array}$ & Local transmission, public transport & Imported, Pakistan \\
\hline Preexisting medical illness & None & None & $\begin{array}{l}\text { Type } 2 \text { diabetes mellitus, } \\
\text { hypertension, and dyslipidemia }\end{array}$ \\
\hline Symptoms and signs & Fever and myalgia & $\begin{array}{l}\text { Fever, anosmia, crepitation right } \\
\text { lung, and deoxygenation }\end{array}$ & $\begin{array}{l}\text { Cough, wheezing both lungs, and } \\
\text { deoxygenation }\end{array}$ \\
\hline Chest radiography at diagnosis & $\begin{array}{l}\text { Alveolar infiltration of the RLL } \\
\text { field }\end{array}$ & Ground-glass infiltration at RLL & $\begin{array}{l}\text { No pulmonary infiltration on } \\
\text { admission } \\
\text { Ground-glass infiltration at both } \\
\text { lower lung fields at day } 3 \text { of } \\
\text { admission }\end{array}$ \\
\hline Diagnosis (day after onset) & 3 & 8 & 3 \\
\hline rRT-PCR for respiratory pathogens & Negative & $\begin{array}{l}\text { H. influenzae in both NPS/TS and } \\
\text { sputum }\end{array}$ & $\begin{array}{l}\text { H. influenzae in both NPS/TS and } \\
\text { sputum }\end{array}$ \\
\hline $\begin{array}{l}\text { Duration from diagnosis to negative } \\
\text { rRT-PCR for SARS-CoV-2 in } \\
\text { NPS/TS vs. sputum (days) }\end{array}$ & 14 vs. 18 & 12 vs. 12 & 27 vs. 40 \\
\hline
\end{tabular}

${ }^{*}$ A full description of case 1 has been reported in ref. 13.

illness. $^{12}$ This discrepancy might be explained by the difference in severity of COVID-19 with moderate severity in our cases and mild cases in the previous study. ${ }^{12}$ Thus, these preliminary results suggested that sputum should be the specimen of choice for the diagnosis and follow-up of COVID19 pneumonia. Self-collection of sputum is simple expectoration, less invasive, and minimizes the risk of transmission to healthcare workers, especially in settings with limited personal protective equipment.

Although the data from this relatively small sample size might limit the ultimate implications of this study, the strong relation between clinical and laboratory findings mirrors the unique feature of this report. However, virus isolation in cell cultures was not performed in this study, so it does not imply that the positive SARS-CoV-2 by rRT-PCR is still infectious.

Received May 28, 2020. Accepted for publication June 26, 2020.

Published online July 8, 2020.

Acknowledgments: We would like to thank our patients and the staff of the Hospital for Tropical Diseases and the Tropical Medicine Diagnostic Reference Laboratory, Faculty of Tropical Medicine, Mahidol University, Bangkok, Thailand. We would like to express our deepest gratitude to Srisin Khusmith for her invaluable suggestions. Publication charges for this article were waived due to the ongoing pandemic of COVID-19.
A

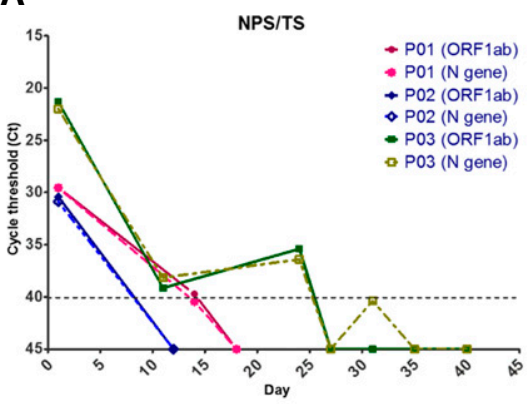

B

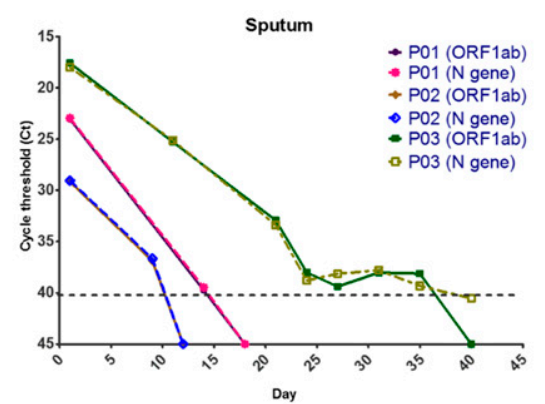

E

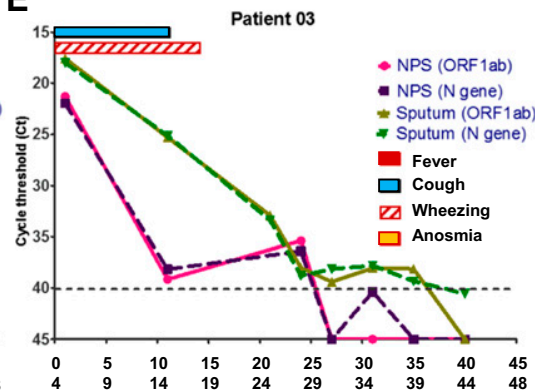

C

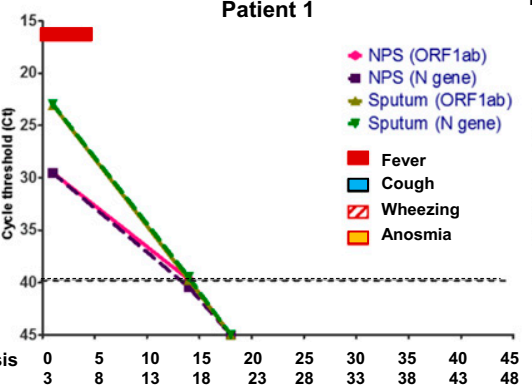

D

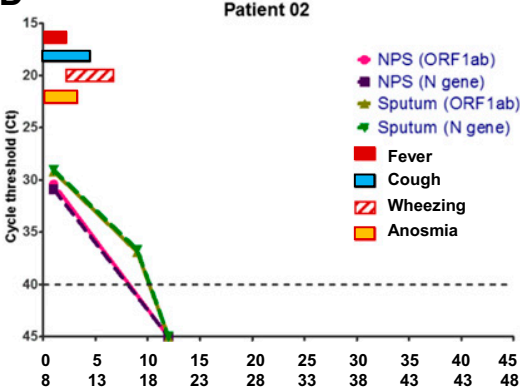

FIGURE 1. Detection results of respiratory specimens by real-time reverse transcriptase-PCR (rRT-PCR) during the clinical course of COVID-19 pneumonia. The concentrations of SARS-CoV RNA in clinical specimens are plotted by the cycle threshold value from nasopharyngeal and throat swabs (A) and sputum (B). The revolution in clinical and rRT-PCR results of the patients 1-3 (C-E). 
Financial support: This work was granted by the Faculty of Tropical Medicine, Mahidol University, Bangkok, Thailand.

Authors' addresses: Viravarn Luvira and Sant Muangnoicharoen, Department of Clinical Tropical Medicine, Faculty of Tropical Medicine, Mahidol University, Bangkok, Thailand, E-mails: viravarn.luv@ mahidol.ac.th and sant.mua@mahidol.ac.th. Akanitt Jittmittraphap and Pornsawan Leaungwutiwong, Department of Microbiology and Immunology, Faculty of Tropical Medicine, Mahidol University, Bangkok, Thailand, E-mails: akanitt.jit@mahidol.ac.th and pornsawan. lea@mahidol.ac.th. Nantarat Chantawat and Weena Janwitthayanan, Tropical Medicine Diagnostic Reference Laboratory, Faculty of Tropical Medicine, Mahidol University, Bangkok, Thailand, E-mails: nantarat.cha@mahidol.ac.th and weena.jan@mahidol.ac.th.

This is an open-access article distributed under the terms of the Creative Commons Attribution (CC-BY) License, which permits unrestricted use, distribution, and reproduction in any medium, provided the original author and source are credited.

\section{REFERENCES}

1. Huang $C$ et al., 2020. Clinical features of patients infected with 2019 novel coronavirus in Wuhan, China. Lancet 395: 497-506.

2. Guan WJ et al.; China Medical Treatment Expert Group for COVID19, 2020. Clinical characteristics of coronavirus disease 2019 in China. N Engl J Med 382: 1708-1720.

3. World Health Organization, 2020. Novel Coronavirus (2019-nCoV) Situation Report -51. Available at: https://www.who.int/docs/ default-source/coronaviruse/situation-reports/20200311sitrep51-covid-19.pdf?sfvrsn=1ba62e57_10. Accessed April 25, 2020.

4. Tang YW, Schmitz JE, Persing DH, Stratton CW, 2020. The laboratory diagnosis of COVID-19 infection: current issues and challenges. J Clin Microbiol 58: e00512-e00520. https:// doi.org/ 10.1128/JCM.00512-20.
5. Clinical Management Oversight Committee, Department of Medical Services Thai Ministry of Public Health COVID-19, 2020. Guidelines for Clinical Practice, Diagnosis, Treatment and Prevention of Healthcare-Associated Infection in Response to Patients with COVID-19 Infection. Thailand Department of Medical Services, Ministry of Public Health. Available at: https://ddc.moph.go.th/viralpneumonia/eng/file/guidelines/g_ CPG.pdf.

6. Rawson TM et al., 2020. Bacterial and fungal co-infection in individuals with coronavirus: a rapid review to support COVID-19 antimicrobial prescribing. Clin Infect Dis ciaa530. https://doi.org/ 10.1093/cid/ciaa530.

7. Kim D, Quinn J, Pinsky B, Shah NH, Brown I, 2020. Rates of coinfection between SARS-CoV-2 and other respiratory pathogens. JAMA 323: 2085-2086.

8. Cuadrado-Payan E et al., 2020. SARS-CoV-2 and influenza virus co-infection. Lancet 395: e84.

9. Wang W, Xu Y, Gao R, Lu R, Han K, Wu G, Tan W, 2020. Detection of SARS-CoV-2 in different types of clinical specimens. JAMA 323: $1843-1844$.

10. Lin C, Xiang J, Yan M, Li H, Huang S, Shen C, 2020. Comparison of throat swabs and sputum specimens for viral nucleic acid detection in 52 cases of novel coronavirus (SARS-Cov-2)infected pneumonia (COVID-19). Clin Chem Lab Med 58: 1089-1094.

11. Yu F et al., 2020. Quantitative detection and viral load analysis of SARS-CoV-2 in infected patients. Clin Infect Dis. https:// doi.org/10.1093/cid/ciaa345.

12. Wolfel R et al., 2020. Virological assessment of hospitalized patients with COVID-2019. Nature 581: 465-469.

13. Nunthavichitra S, Prapaso S, Luvira V, Muangnoicharoen S, Leaungwutiwong P, Piyaphanee W, 2020. Case report: COVID19 presenting as acute undifferentiated febrile illness-A tropical world threat. Am J Trop Med Hyg 103: 83-85. 